\title{
Complicações cardiovasculares em pacientes com Diabetes Mellitus Tipo 2
}

\author{
Cardiovascular complications in patients with Type 2 Diabetes Mellitus
}

Complicaciones cardiovasculares en pacientes con Diabetes Mellitus Tipo 2

Maria Angélica Otero de Melo dos Reis ${ }^{1 *}$, Antônio Bosi Castro de Oliveira ${ }^{2}$, Bárbara Camargo Levy $^{3}$, Fernanda Marinho de Souza ${ }^{4}$, Giovana Nunes de Assunção ${ }^{5}$, Isabele Dória Cabral Correia $^{5}$, Júlia Morais de Moura ${ }^{6}$, Maryana Duarte Costa ${ }^{7}$, Thayna de Andrade Romeu Alexandre $^{7}$, Guilherme Neif Vieira Musse ${ }^{8}$.

\section{RESUMO}

Objetivo: O presente artigo buscou realizar uma revisão acerca das complicações microvasculares e macrovasculares em pacientes com Diabetes Mellitus Tipo 2 (DMT2), bem como esclarecer o comportamento do corpo humano frente a essas alterações e importância do diagnóstico e tratamento precoce para uma melhor qualidade de vida desses indivíduos. Revisão bibliográfica: A DMT2 é uma doença em crescimento alarmante que contribui principalmente para o desenvolvimento de complicações cardiovasculares. As complicações do DMT2 incluem Doença Renal Diabética, Retinopatia Diabética, Neuropatia Diabética e Disfunção Sexual, sendo estas microvasculares. Observa-se ainda a ocorrência de Insuficiência cardíaca, Derrame e Infarto Agudo do Miocárdio como as principais complicações macrovasculares. Essas alterações estão diretamente relacionadas à Hipertensão Arterial Sistêmica, Dislipidemias e Obesidade, ocasionando uma alta letalidade, principalmente quando relacionada à insuficiência cardíaca nesses indivíduos. Considerações finais: Verificou-se neste trabalho que o comprometimento da qualidade de vida pelo DMT2 reduz a expectativa de vida dos indivíduos quando comparados aos não diabéticos.

Palavras-chave: Angiopatia diabética, Diabetes, Cardiologia.

\begin{abstract}
Objective: This article sought to conduct a review on microvascular and macrovascular complications in patients with Type 2 Diabetes Mellitus (DMT2), as well as to clarify the behavior of the human body in the face of these changes and the importance of early diagnosis and treatment for a better quality of life of these individuals. Bibliographic review: DMT2 is an alarmingly growing disease, which mainly contributes to the development of cardiovascular complications. The complications of DMT2 include Diabetic Renal Disease, Diabetic Retinopathy, Diabetic Neuropathy and Sexual Dysfunction, these being microvascular. It is also observed the occurrence of Heart Failure, Stroke and Acute Myocardial Infarction as the main macrovascular complications. These alterations are directly related to Systemic Arterial Hypertension, Dyslipidemias and Obesity, causing a high lethality mainly when related to heart failure in these individuals. Final considerations: It was verified in this work that the impairment of quality of life by DMT2 reduces the life expectancy of individuals when compared to non-diabetics.
\end{abstract}

Keywords: Diabetic angiopathy, Diabetes, Cardiology.

\footnotetext{
1 Universidad Nacional de Rosaio (UNR), Rosario - Santa Fé, Argentina.

*E-mail: mariaoteromeloreis@gmail.com

2 Instituto Metropolitano de Ensino Superior (UNIVAÇO), Ipatinga - MG.

3 Universidade Cidade de São Paulo (UNICID), São Paulo - SP.

${ }^{4}$ Universidade do Estado da Bahia (UNEB), Salvador - BA.

${ }^{5}$ Universidade Potiguar (UnP), Natal - RN.

${ }^{6}$ Universidade Federal do Estado do Rio de Janeiro (UNIRIO), Rio de Janeiro - RJ.

${ }^{7}$ Centro Universitário Presidente Antônio Carlos (UNIPAC), Juiz de Fora - MG.

8 Universidade Federal de Juiz de Fora (UFJF), Juiz de Fora - MG.
} 


\section{RESUMEN}

Objetivo: El objetivo de este artículo era realizar una revisión de las complicaciones micro y macrovasculares en pacientes con diabetes mellitus tipo 2 (DMT2), así como aclarar el comportamiento del cuerpo humano ante estos cambios y la importancia del diagnóstico y el tratamiento tempranos para una mejor calidad de vida de estos individuos. Revisión bibliográfica: EI DMT2 es una enfermedad de alarmante crecimiento, que contribuye principalmente al desarrollo de complicaciones cardiovasculares. Las complicaciones del DMT2 incluyen la enfermedad renal diabética, la retinopatía diabética, la neuropatía diabética y la disfunción sexual, que son microvasculares. También se observa la aparición de la insuficiencia cardíaca, el derrame cerebral y el infarto agudo de miocardio como las principales complicaciones macrovasculares. Estas alteraciones están directamente relacionadas con la hipertensión arterial sistémica, las dislipidemias y la obesidad, causando una alta tasa de mortalidad, especialmente cuando se relacionan con la insuficiencia cardíaca en estos individuos. Consideraciones finales: En este estudio se verificó que el deterioro de la calidad de vida por el DMT2 reduce la esperanza de vida de los individuos en comparación con los no diabéticos.

Palabras clave: Angiopatía diabética, Diabetes, Cardiología.

\section{INTRODUÇÃO}

O Diabetes Mellitus (DM) é uma doença metabólica que apresenta como característica principal o aumento da glicose no sangue (hiperglicemia), causada por uma deficiência na produção de insulina pelo organismo. O DM se diferencia em dois tipos: o diabetes mellitus tipo 2 (DMT2), mais comum e profundamente influenciada por fatores externos, enquanto o diabetes mellitus tipo 1 (DMT1) tem origem predominantemente genética e hereditária (SLIECKER RC, et al., 2019; LI S, et al., 2019).

É importante ressaltar que devido a seus sintomas, em sua maioria silenciosa, é comum que os pacientes recebam diagnóstico tardio, quando a doença já se apresenta em fase avançada e podendo levar a consequências irreversíveis. O DM está associado a várias comorbidades, com complicações macrovasculares e microvasculares, bem como a condicões crônicas como hipertensão, síndrome metabólica (SM) e doenças cardiopulmonares, o que torna o tratamento e o controle da doença indispensável (INNANIR $\mathrm{M}$, et al., 2019).

Dentre as complicações macrovasculares, as mais comuns são as doenças cardiovasculares (DCV), tais como: insuficiência cardíaca, derrame e infarto (HUANG, et al., 2017). Quanto às alterações microvasculares, destaca-se a retinopatia diabética (RPD), neuropatia diabética (NPD), doença renal diabética (DRD) e disfunção sexual (DS) (COLE JB e FLOREZ JC, 2020). Assim, faz-se necessário a suspeita clínica e reconhecimento precoce destas doenças, uma vez que uma grande parte dos pacientes já apresenta uma ou mais complicações, mesmo com o DMT2 recém diagnosticado (FASELIS C, et al., 2020).

Além disso, acredita-se que existe uma tendência à ocorrência simultânea de ambas as complicações vasculares em pacientes diabéticos (ROY S, et al.,2020; COLE JB e FLOREZ JC, 2020). Diabetes mellitus é um agravante para doença cardiovascular aterosclerótica (ASCVD), sendo responsável pela maioria das mortes cerebrovasculares por doença cardíaca isquêmica e celular.

Senso assim, o objetivo desse estudo foi analisar os riscos relacionados às complicações cardiovasculares em pacientes que apresentam DMT2, visto que é comum a existência de pacientes com diabetes descompensado e sem controle, ou até não diagnosticado, fato que pode gerar desde as alterações glicêmicas comuns da doença às complicações severas e, em vários casos, irreversíveis.

\section{REVISÃO BIBLIOGRÁFICA}

Cerca de 347 milhões de pessoas no mundo apresentam DM (GORE M, et al., 2015). De acordo com a Organização Mundial da Saúde (OMS), o número de pessoas portadoras aumentou em 314 milhões comparando os anos de 1980 e 2014. Da mesma forma, elevaram-se os índices de adultos com mais de 18 anos que apresentam DM, passando de 4,7\% em 1980 para 8,5\% em 2014 (ROY S, et al., 2020). Expectativas apontam que para 2040, são estimados mais de 640 milhões de pacientes diabéticos na população adulta do mundo (FASELIS C, et al., 2020). 
O aumento alarmante se dá, principalmente, pela grande incidência de DMT2 e obesidade, sendo uma grave crise de saúde pública (GORE M, et al., 2015; HAN D, et al., 2019), além disso, está entre as 5 principais causas de mortalidade na maioria dos países de alta renda e, portanto, é um fardo no sistema de saúde pública (HACKETT RA e STEPTOE A, 2016).

Nesse sentido, o DMT2 é considerado o principal fator de risco para danos nos sistemas macrovasculares e microvasculares (GORE M, et al., 2015; COLE JB e FLOREZ JC, 2020). Representa cerca de 90\% a 95\% de todos os casos de DM (FASELIS C, et al., 2020). Estimativas evidenciam o risco de duas a quatro vezes maior de um indivíduo diabético desenvolver complicações cardiovasculares, comparado a um indivíduo não diabético (ROY S, et al., 2020; GORE M, et al., 2015; HACKETT RA e STEPTOE A, 2016; PARHOFER KG, et al., 2019). Estudos apontam que pacientes com DM morrem, em média, 6 anos mais cedo quando comparados com pacientes sem DM (HACKETT RA e STEPTOE A, 2016).

As doenças cardíacas e vasculares afetam os indivíduos com DMT2 de formas distintas, apresentando uma variabilidade multifatorial (GORE M, et al., 2015). Os fatores que estão relacionados ao risco vascular e possibilitam a heterogeneidade das DCV são: idade, sexo, etnia, classe econômica, disponibilidade de serviços de saúde, medicamentos, genética, hábitos comportamentais, comorbidades e variabilidade dos níveis de glicemia (ROY S, et al., 2020; GORE M, et al., 2015; COLE JB e FLOREZ JC, 2020; SLIECKER RC, et al., 2019; FASELIS C, et al., 2020; HACKETT RA e STEPTOE A, 2016).

Hábitos diários, como sedentarismo e má alimentação, também intensificam o risco cardiovascular em diabéticos visto que resultam em alta prevalência de sobrepeso e obesidade, e na elevação das taxas de ácidos graxos e colesterol. Além disso, observa-se a presença de comorbidades como hipertensão e hipertensão resistente ao tratamento (CARDOSO CRL, et al., 2019), dislipidemia, doenças renais, distúrbios do sono e depressão (GORE M, et al., 2015; HACKETT RA e STEPTOE A, 2016).

Por fim, a glicemia alta afeta as vias de sinalização celular, através do aumento da produção de substâncias reativas ao oxigênio, promovendo a ocorrência do estresse oxidativo. Consequentemente, ocorrem modificações na expressão gênica, apoptose de células do músculo cardíaco e disfunção de vasos sanguíneos, o que contribui para a incidência de DCV (ROY S, et al., 2020; COLE JB e FLOREZ JC, 2020). Logo, reforça-se a importância do correto manejo desses fatores, visando a redução de complicações microvasculares e macrovasculares em indivíduos com DMT2 (MARTINS EB, et al., 2020).

O DM é uma condição que expõe o paciente a um risco excessivo de mortalidade cardiovascular e por todas as causas por estar fortemente associada às mortes por doenças cerebrovasculares e doenças isquêmicas do coração (TANG O, et al., 2019; CARDONA A, et al., 2019). Pesquisa realizada por Tang O, et al. (2019) constatou que a taxa de mortalidade cardiovascular foi significativamente maior entre os pacientes com diabetes de longa data quando comparados àqueles sem diabetes ou com diabetes de curta duração.

Além disso, já foi demonstrado que, de que na presença de DCV, os diabéticos têm resultado e progressão piores (HAN D, et al., 2019), ou seja, à medida que aumenta a duração da doença, os riscos de complicações microvasculares e macrovasculares também se elevam (TANG O, et al., 2019; HUANG, et al., 2017). Consequentemente, estes indivíduos têm piores resultados após evento cardiovascular (ECV), risco aumentado para recorrência de Acidente Vascular Cerebral (AVC) e redução da recuperação cognitiva e funcional (HACKETT RA e STEPTOE A, 2016; DESAI D, et al., 2015).

O controle da glicose não deve ser o único alvo do tratamento do paciente com DM, uma vez que os resultados advindos da redução intensa dos níveis de glicose tiveram descrições heterogêneas em alguns estudos (LI S, et al., 2019). Apesar do rígido controle da glicose ser relevante para a redução do risco de ECV e das complicações microvasculares do DM, o estudo ACCORD que avaliou o controle rígido da glicose e seus efeitos, apresentou uma adversidade: aumento significativo da mortalidade e eventos cardiovasculares nos pacientes que o fizeram (LI S, et al., 2019; CARDONA A, et al., 2019, HUANG, et al., 2017). Dentre os fatores que podem explicar a diferença entre os desfechos cardiovasculares, se encontra a variabilidade glicêmica (LI S, et al., 2019; HUANG, et al., 2017). 
A redução do risco de tais desfechos em pacientes com DM está atrelada às mudanças no estilo de vida e ao uso correto de medicamentos. Além disso, devido ao aumento da prevalência do DM2, se faz necessário a identificação dos mecanismos de DCV associadas ao DM2 (CARDONA A, et al., 2019; MARTINS EB, et al., 2020; HUANG, et al., 2017).

\section{Doença Renal Diabética}

A Doença Renal Diabética (DRD) é caracterizada pela redução progressiva da filtração glomerular renal causada pela hiperglicemia e pela albuminúria. Entretanto, a albuminúria pode ocorrer devido a outros fatores, como infecções. Outra modalidade de diagnóstico se dá através da biópsia renal, que é o padrão ouro para diagnóstico de DRD, mas é raramente realizada, sendo somente feita em casos nos quais outras patologias possam estar envolvidas (COLE JB e FLOREZ JC, 2020; FASELIS C, et al., 2020).

A doença renal em indivíduos com DMT2 pode ser ocasionada por fatores de riscos independentes, ou indiretamente relacionados ao diabetes, como é o caso da hipertensão, obesidade ou dislipidemia (COLE JB e FLOREZ JC, 2020). A DRD não possui cura, entretanto, o tratamento se dá por meio da manutenção da estabilidade dos níveis de glicemia e albuminúria através do uso de medicamentos, como anti-hipertensivos, e pelo acompanhamento do comprometimento renal.

Nos estágios finais da doença, o paciente é submetido a sessões de diálise ou é realizado um transplante renal para manutenção da vida (COLE JB e FLOREZ JC, 2020). A DRD é a causa mais frequente em pacientes no estágio terminal de doenças renais e é uma comorbidade que aumenta em duas vezes os riscos cardiovasculares, já elevados pelos motivos discutidos anteriormente (GORE M, et al., 2015; FASELIS C, et al., 2020).

\section{Neuropatia Diabética}

A Neuropatia Diabética (NPD) afeta, pelo menos, metade dos pacientes com mais de 10 anos de doença e até $20 \%$ dos pacientes no momento do diagnóstico. Entretanto, apesar da alta prevalência, algumas vezes o diagnóstico desse quadro pode passar despercebido, visto que até $50 \%$ dos pacientes são assintomáticos. A NPD é composta por diversas manifestações clínicas e por isso deve ser feito o diagnóstico de exclusão para disfunção do nervo periférico (FASELIS C, et al., 2020). Os nervos periféricos longos de membros inferiores são frequentemente acometidos pelo DM, principal causa de desenvolvimento de danos em nervos (COLE JB e FLOREZ JC, 2020).

A NPD pode ser classificada de acordo com a prevalência, distribuição anatômica, curso clínico, características e fisiopatologia (FASELIS C, et al., 2020). As neuropatias periféricas diabéticas podem ser divididas em categorias, tais como: neuropatias autônomas, neuropatias atípicas, neuropatias não diabéticas comuns no DM e, por fim, a forma mais comum de NPD que é a polineuropatia simétrica distal (COLE JB e FLOREZ JC, 2020; FASELIS C, et al., 2020).

O diagnóstico da NPD é clínico, uma vez que o paciente apresenta sintomas característicos do acometimento das fibras grandes ou pequenas. $O$ envolvimento das fibras pequenas está relacionado com a presença de dor com piora noturna, disestesia, hiperalgesia e alodínia (COLE JB e FLOREZ JC, 2020). Por outro lado, o acometimento de fibras grossas é responsável pelo aparecimento de dormência e perda de sensibilidade. A avaliação do indivíduo diabético para a NPD deve ser feita no momento do diagnóstico e nos anos seguintes, semelhante ao que ocorre com as outras complicações (FASELIS C, et al., 2020).

Essa complicação microvascular pode evoluir com sérias consequências, caso não seja reconhecida e devidamente tratada (FASELIS C, et al., 2020). Dentre as complicações da NPD têm-se a amputação de membros e desenvolvimento de úlcera de pé e neuroartropatia de Charcot, sendo que todas afetam significativamente a qualidade e expectativa de vida (COLE JB e FLOREZ JC, 2020; FASELIS C, et al., 2020).

É importante ressaltar que o tratamento é sintomático e totalmente dependente da prevenção a partir do controle glicêmico (COLE JB e FLOREZ JC, 2020). Atualmente não existe nenhum tratamento capaz de reverter ou impedir a progressão da doença (FASELIS C, et al., 2020). 


\section{Retinopatia Diabética}

A Retinopatia Diabética (RPD) é atualmente a principal causa de cegueira associada ao diabetes no mundo. Essa doença pode ser classificada em uma forma não proliferativa precoce caracterizada por vasos sanguíneos enfraquecidos ou na forma proliferativa, mais grave e tardia, caracterizada pelo crescimento de novos vasos sanguíneos frágeis e vazados em toda a retina e no vítreo (JOANNE B e FLOREZ C, et al., 2020).

$\mathrm{Na}$ RPD, a perda de visão é geralmente atribuída ao edema que prejudica a visão central ou, no caso da RPD proliferativa, pode levar à formação de novos vasos e tecido fibroso no disco ou em outros lugares na retina, resultando em descolamento fracionado da retina e hemorragia pré retiniana, levando à formação de lesões mais graves.

Os fatores de risco para o desenvolvimento da RPD são o controle glicêmico e controle da pressão arterial inadequados e a dislipidemia. Nesse sentido, o estudo UKPDS comprovou que o controle glicêmico intensivo resultou em uma diminuição de risco de $35 \%$ na necessidade de fotocoagulação, de $34 \%$ na taxa de progressão da RPD e $47 \%$ na deterioração da atividade visual.

Para um diagnóstico preciso, todos os pacientes devem ser cuidadosamente avaliados por um oftalmologista para detectar sinais precoces de RPD, que geralmente são assintomáticos. Para tanto, a Oftalmoscopia em fundo dilatado e a fotografia de fundo estereoscópica de 7 campos fornecem dados minuciosos para a detecção e estadiamento da doença. $O$ ideal é que o exame seja realizado em dois anos consecutivos e, caso não apresente evidências positivas da doença e exista um bom controle glicêmico, seja repetido em intervalos de tempo mais amplos, como a cada 3 anos, segundo a American Diabetes Association 2018 (ADA) (FASELIS C, et al., 2020).

\section{Disfunção Sexual}

A Disfunção Sexual (DS) é uma complicação em pacientes com DMT2 considerada irrelevante, mesmo com seu evidente impacto na autoestima e qualidade de vida dos pacientes. Ela é resultado de alterações vasculares, neuronais e hormonais consequentes da DM. A DS e a doença arterial coronariana (DAC) estão relacionadas pois a presença de DS indica o desenvolvimento de DAC em grandes artérias, origem do acometimento vascular, podendo assim servir como triagem para prevenção, principalmente em pacientes jovens (FASELIS C, et al., 2020; IMPRIALOS KP, et al., 2018).

No caso dos homens, a DS é caracterizada pela dificuldade de conseguir ou manter a ereção peniana para a relação sexual. Já nas mulheres, corresponde a diminuição do desejo sexual, da excitação sexual, presença de dor durante ou após o ato sexual e incapacidade de chegar ao orgasmo. Esses sintomas, tanto no homem quanto nas mulheres, devem permanecer por no mínimo 3 meses para que o diagnóstico seja feito (FASELIS C, et al., 2020).

As comorbidades atreladas a DM, como hipertensão, dislipidemia, depressão e DCV, colaboram para o desenvolvimento da DS em pessoas diabéticas. Nos homens, a ingestão de medicamentos anti-hipertensivos antigos, como betabloqueadores e diuréticos, e agentes antidepressivos podem ser a causa da DS (IMPRIALOS KP, et al., 2018). No entanto, a prática de exercícios físicos, lazer e consumo de uma baixa quantidade de álcool colaboram positivamente, evitando os sintomas de DS (FASELIS C, et al., 2020). Com relação às mulheres, não há informações suficientes para determinação dos fatores de risco e causas (MASEROLI E, et al., 2018).

\section{Dislipidemia}

Sabe-se que o colesterol é um composto orgânico químico lipídico, derivado dos álcoois, que agrega a membrana das células do organismo humano. O aumento das concentrações circulantes de glicose e ácidos graxos livres leva à deposição lipídica inadequada em tecidos extra porosos, incluindo o coração. No entanto, os cardiomiócitos são insuficientemente equipados para armazenar grandes quantidades de lipídios com acilgliceróis acumulados e ceramidas, causando danos celulares por lipotoxicidade (LEHRKE M e MARX N, 2017; PARHOFER KG, et a., 2019). 
O fenótipo lipídico aterogênico presente na SM é resultado do excesso de tecido adiposo visceral que, devido à intensa atividade lipolítica, libera grandes quantidades de ácidos graxos livres na circulação. Em consequência, há menor depuração hepática de insulina, resultando em hiperinsulinemia e uma maior secreção hepática de lipoproteínas de muito baixa densidade (VLDL) (STAHLIL BE, et al., 2015). Consequentemente, isso resulta em maior geração de lipoproteínas de baixa densidade (LDLs), que são menores e mais densas, diminuição do colesterol da lipoproteína de alta densidade (HDL) e o aumento dos triglicérides. Nesse sentido, pode-se associar o maior grau de dislipidemia a pacientes diabéticos que têm maior risco de DCV, pois a aplicação do HDL modifica a partícula, aumenta sua carga negativa e facilita sua oxigenação, tornando-a mais aterogênica (PEREL C, 2018).

Outra comorbidade de alto impacto é a remodelagem concêntrica ventricular esquerda como uma característica relevante do miocárdio diabético, decorrente da deposição de triglicérides e aumento do volume extracelular, que resulta em deposição de colágeno e fibrose, sendo preditivo para mortalidade e insuficiência cardíaca (PEREL C, 2018). A remodelagem ocorre, pois, o aumento e consequente da rigidez do miocárdio traduz-se em disfunção diastólica, redução da tensão do miocárdio e alargamento atrial, que tem sido associado a uma maior prevalência de fibrilação atrial em pacientes com diabetes (LEHRKE M e MARX N, 2017).

\section{Doenças Cardíacas}

As Doenças Cardíacas (DACs) são uma das principais causas de morte e morbidade em diabéticos. Como foi relatado anteriormente, o diabetes está associado a várias comorbidades, como hipertensão, doenças cardiovasculares, síndrome metabólica e doenças cardiopulmonares. Durante um período de tempo prolongado, o DM é também um importante fator de risco subjacente para doença cardíaca coronária, insuficiência cardíaca, doença arterial periférica, fibrilação atrial e derrame (INANIR M et al., 2020). O aumento do risco cardiovascular em indivíduos portadores de diabetes mellitus não é totalmente explicado por esses riscos clássicos, mas diretamente relacionado com a hiperglicemia (SLIECKER RC, et al.,2019).

Diante desse contexto, torna-se evidente que o controle hiperglicêmico é a principal razão para evitar complicações angiopáticas e seu controle deficiente pode desempenhar um papel significativo no desenvolvimento de doenças cardiovasculares em diabéticos (DESAI D, et al., 2015). Além do controle da hiperglicemia, o procedimento de revascularização do miocárdio para o tratamento de doença coronariana (DAC) em pacientes com DM tem-se mostrado benéfico no alívio dos sintomas e como um importante fator de sobrevida (EBRAHIM MEBM, et al., 2019).

Ao relacionar-se as quantidades de lipídios circulantes no sangue em conjunto com o distúrbio metabólico já mencionado, o aumento de lipídeos nos cardiomiócitos gera uma reação de dano celular por lipotoxicidade, o que leva a uma reação inflamatória, que amplia a resistência à insulina. A disfunção cardíaca existente entre a elevação da glicose e a presença de ácidos graxos que alteram cadeias bioquímicas existentes para a produção de energia e há produção de espécies reativas de oxigênio, sendo uma patologia central da complicação de diabetes no aparelho cardiovascular (LEHRKE M e MARX N, 2017; PEREL C, 2018).

Vale ressaltar que a hipertrofia do coração dos diabéticos está relacionada com a deposição de triglicerídeos no miocárdio e também com o aumento da deposição de colágeno. Em estudos, foram encontradas combinações entre a disponibilidade de oxigênio e sua perfusão do miocárdio, como também a disponibilidade energética, que geram produtos finais que determinam danos microvasculares, associados à rigidez de cardiomiócitos, contribuindo para cardiomiopatia diabética (LEHRKE M e MARX N, 2017).

Para os portadores de diabetes mellitus, são necessários exames rotineiros para prevenção, principalmente, de doenças macrovasculares, que afetam o sistema cardíaco. Para isso, um tipo de exame necessário é a utilização de eletrocardiogramas (ECG) (INANIR M, et al., 2020).

Estudos realizados entre janeiro de 2017 e maio de 2018, foi possível verificar que a frequência cardíaca, relações Tp-e (intervalo de tempo entre o pico e o final da onda T) / QT (medida dada pelo início do complexo QRS até o final da onda T) e Tp-e/QTc ( QT corrigido) e os intervalos QTc, QTd (disperção do intervalo QT), QTdc (dispersão do intervalo QT corrigido), Tp-e e JTc (JT corrigido, sendo que JT é o intervalo de tempo 
entre o final do complexo QRS até o final da onda T), foram significativamente maiores quando comparado aos pacientes do grupo de controle e também, avaliados os ECGs dos pacientes com diabetes, obteve-se como resultado a repolarização prolongada quando comparado com os demais, que relacionado com as arritmias ventriculares e morte súbita (INANIR M, et al., 2020).

Para comprovar a tese de que DCV são mais letais em pacientes com DM, um estudo analisou pacientes com insuficiência cardíaca portadores ou não de DM. O estudo Dinamarquês de Investigações de Arritmia e Mortalidade com Dofetilide (DIAMOND) analisou a influência do diabetes no risco de morte em 5.491 pacientes hospitalizados que possuíam insuficiência cardíaca congestiva quando acompanhados por 5 a 8 anos (GUSTAFSSON I, et al., 2004 apud LEHRKE M e MARX N, 2017).

Foi constatado que, entre os analisados, $16 \%$ dos pacientes tinham diabetes no início do estudo e aproximadamente $50 \%$ tinham uma fração de ejeção $<35 \%$, sugerindo que na subpopulação haviam pacientes com fração de ejeção preservada e não preservada. Nesse mesmo estudo, em análises mais elaboradas de mortalidade, observou-se que de $31 \%$ a $50 \%$ dos pacientes com insuficiência cardíaca portadores de diabetes foram a óbito após três anos do estudo (GUSTAFSSON I, et al., 2004 apud LEHRKE M e MARX N, 2017).

\section{CONSIDERAÇÕES FINAIS}

Diante do exposto, é possível concluir que o paciente com DMT2 pode evoluir para diversas complicações macro e microvasculares, sendo a insuficiência cardíaca a mais letal entre esses indivíduos. O DM pode se apresentar de maneira silenciosa, entretanto, suas complicações possuem alto nível de mortalidade e morbidade. Atualmente, muitas dessas complicações como a cegueira diabética e a neuropatia diabética, não possuem cura e afetam diretamente a qualidade de vida do paciente. Logo, as evidências crescentes demonstram um aumento alarmante na incidência de pessoas com DM, sendo, imprescindível que o profissional da saúde fique atento a todas possíveis complicações e dê importância e prioridade nas políticas e ações de prevenção da doença em suas distintas formas e na promoção de hábitos de vida saudáveis.

\section{REFERÊNCIAS}

1. CARDONA A, et al. Trimethylamine N-oxide and incidente atherosclerotic events in high-risk individuals with diabetes: and ACCORD trial post hoc analysis. BMJ Open Diabetes Research and Care, 2019; 7:e000718.

2. CARDOSO CRL, et al. Prognostic Importance of Resistant Hypertension in Patients With Type 2 Diabetes: The Rio de Janeiro Type 2 Diabetes Cohort Study. Diabetes Care, 2020; 43(1): 219-227.

3. COLE JB, FLOREZ JC. Genetics of diabetes mellitus and diabetes complications. Nature Reviews Nephrology, 2020; 16: 377-390.

4. DESAI D, et al. Preventing Cardiovascular Disease in Patients with Diabetes: Use of Aspirin for Primary Prevention. Curr Cardiol Rep, 2015. 17(13).

5. EBRHAIM MEBM, et al. Late Clinical Outcomes of unselected patients with diabetic mellitus and multi-vessel coronary artery disease. International Journal of Cardiology, 2019; 296(1): 21-25.

6. FASELIS C, et al. Microvascular Complications of Type 2 Diabetes Mellitus. Current Vascular Pharmacology, 2020; 18(2): 117-124.

7. GORE MO, et al. Predicting Cardiovascular Risk in Type 2 Diabetes: the Heterogeneity Challenges. Curr Cardiol Rep, 2015. 17(54).

8. HACKETT RA, STEPTOE A. Psychosocial Factors in Diabetes and Cardiovascular Risk. Current Cardiology Reports, 2016; 18(10): 95.

9. HAN D, et al. Myocardial Ischemic Burden and Differences in Prognosis Among Patients With and Without Diabetes: Results From the Multicenter International REFINE SPECT Registry. Diabetes Care, 2020; 43(2): 453-459.

10. HUANG D, et al. Macrovascular Complications in Patients with Diabetes and Prediabetes. BioMed Research International, 2017. ID7839101, 9 p.

11. IMPRIALOS KP, et al. Sexual dysfunction, cardiovascular risk and effects of pharmacotherapy. Curr Vasc Pharmacol 2018; 16: 130-42.

12. INANIR M, et al. Evaluation of Electrocardiographic Ventricular Depolarization and Repolarization Variables in Type 1 Diabetes Mellitus. Arq Bras Cardiologia( online), 2020. 114(2): 275-280.

13. LEHRKE M, MARX N. Diabetes Mellitus And Heart Failure. Am J Cardiol, 2017. 120: 37-47.

14. LI S, et al. Visit-to-Visit HbA $\mathrm{H}_{1 \mathrm{c}}$ Variability Is Associated With Cardiovascular Disease and Microvascular Complications in Patients With Newly Diagnosed Type 2 Diabetes. Diabetes Care, 2020; 43(2): 426-432. 
15. MARTINS EB, et al. Pharmacological therapy and cardiovascular risk reduction for type 2 diabetes. Rev Assoc Med Bras, 2020. 66(9): 1283-1288.

16. MASEROLI E, et al. Cardiometabolic risk and female sexuality-part I. Risk factors and potential pathophysiological underpinnings for female vasculogenic sexual dysfunction syndromes. Sex Med Rev. 2018; 6(4): 508-24.

17. PARHOFER KG, et al. Position on Lipid Therapy in Patients with Diabetes Mellitus. German Diabetes Association: Clinical Practice Guidelines. Exp. Clin Endocriol Diabetes, 2019. 127(1): 97-101.

18. PEREL C. Insuficiencia cardíaca y diabetes Nuevos tratamientos para la diabetes. Insuf Card, 2018.13(4):155-169.

19. ROY S, et al. Systolic dysfunction in asymptomatic type 2 diabetic patients, a harbinger of microvascular complications: A cross-sectional study from North India. Diabetes \& Vascular Research, 2017.1(7).

20. SLIECKER RC, et al. Visit-to-visit variability of glycemia and vascular complications: the Hoorn Diabetes Care System cohort. Cardiovasc Diabetal, 2019. 18(170).

21. STÄHLI BE, et al. Lipid Effects and Cardiovascular Disease Risk Associated with Glucose-Lowering Medications. Curr Cardiol Rep, 2015.17(55).

22. TANG O, et al. Mortality Implications of Prediabetes and Diabetes in Older Adults. Diabetes Care, 2020; 43(2): 382388. 